\title{
Addition of Mannitol to Hyaluronic Acid may Shorten Viscosupplementation Onset of Action in Patients with Knee Osteoarthritis: Post-Hoc Analysis of A Double-blind, Controlled Trial
}

\author{
Florent Eymard ${ }^{1}$, Marie Bossert ${ }^{2}$, Roger Lecurieux ${ }^{3}$, Bernard Maillet ${ }^{4}$, Xavier Chevalier ${ }^{1}$ and Thierry \\ Conrozier ${ }^{2 *}$
}

${ }^{1}$ Department of Rheumatology, Hôpital Henri Mondor, Creteil, France

${ }^{2}$ Department of Rheumatology, Hôpital Nord Franche-Comté, Belfort, France

${ }^{3}$ Department of Rheumatology, Hôpital Emile Muller, Mulhouse, France

${ }^{4}$ Department of Rheumatology, Clinique Juge, Marseille, France

${ }^{*}$ Corresponding author: Thierry Conrozier, Department of Rheumatology, Hôpital Nord Franche-Comté, 14 rue de Mulhouse, Belfort, 90000 , France, Tel +33686919218; E-mail: thierry_conrozier@hotmail.fr or thierry.conrozier@hnfc.fr

Received date: July 04, 2016; Accepted date: July 22, 2016; Published date: July 27, 2016

Citation: Eymard F, Bossert M, Lecurieux R, Maillet B, Chevalier X, et al. (2016) Addition of Mannitol to Hyaluronic Acid may Shorten Viscosupplementation Onset of Action in Patients with Knee Osteoarthritis: Post-Hoc Analysis of A Double-blind, Controlled Trial. J Clin Exp Orthop 2: 21. doi: $10.4172 / 2471-8416.100021$

Copyright: (C) 2016 Eymard F, et al. This is an open-access article distributed under the terms of the Creative Commons Attribution License, which permits unrestricted use, distribution, and reproduction in any medium, provided the original author and source are credited.

\section{Abstract}

Objectives: To compare the speed of action of three weekly intra-articular injections of a combination of hyaluronic acid and mannitol (HAnox-M) with that of hyaluronic acid alone (BioHA), in patients with knee osteoarthritis (OA).

Methods: Post-hoc analysis of a randomized, double blind, controlled trial demonstrating the non-inferiority of an association HAnox-M compared to BioHA at month 6 after injections. Data from 205 patients with symptomatic knee OA (Intent-to-Treat population) were retrospectively analyzed. The primary outcome was 1 and 2 week change in the WOMAC pain subscale (0-20). The number and percentage of improved patients at week 1 and 2 were also studied, as well as the level of improvement.

Results: HAnox-M and BioHA groups were not statistically different at baseline and month 6 . The median WOMAC pain score at baseline was 9 in both groups. It was 6.0 and 5.0 in the HAnox-M group at Week 1 and Week 2 respectively. It was 7.0 and 6.0 in the BioHA group, namely a decrease of 1 more point in favor of HANOX, obtained from as soon as the 1st injection. At month 3 and 6 the results were identical (5.0 and 4.0 respectively) for both groups. In subjects with grade 3 joint space narrowing $(N=84)$ the decrease of pain (SD) was significantly greater at week 3 in patients treated with HAnox-M than in those treated with Bio-HA:-4.2 (3.2) versus -2.8 (2.6) respectively $(p=0.048)$.

Conclusion: In patients with symptomatic knee osteoarthritis, addition of mannitol to HA may shorten the onset of action of viscosupplementation, chiefly in patients with advanced stage of the disease.

Keywords: Knee osteoarthritis; Viscosupplementation; Mannitol; Hyaluronic acid; Intra-articular

\section{Introduction}

The medical management of knee osteoarthritis (OA) includes a combination of non-pharmacological and pharmacological modalities [1-4]. Among them, intra-articular (IA) injection(s) of hyaluronic acid (HA) [5] is widely used worldwide to reduce joint pain and improve function in patients not adequately relieved with conventional therapy. The mechanisms of action of viscosupplementation are complex and not yet fully understood. The more probable hypothesis is a transient restoration of the physiological joint homeostasis [6]. Moreover there are increasing in vitro and in vivo evidences that HA could have chondroprotective properties [7-12] whereas metaanalyses continue to show controversial results regarding the efficacy and safety of viscosupplementation [13-17].

One point on which everyone agrees is that that corticosteroids are more effective than HA in the short term (up to 4 weeks), whereas HA becomes more effective from 4 weeks and in the long term (up to 26-52 weeks) [18]. The delayed onset of action of HA leads many physicians to use coadministration of the 2 agents assuming a synergistic effect, useful in clinical practice to alleviate pain quickly. Hasten the onset of action of $\mathrm{HA}$ would be a significant therapeutic advance, the delayed action (up to 8 weeks) of IA HA, being one of the main concern with this treatment. One hypothesis for this delayed effectiveness of viscosupplementation is the limited 
time of residence into the joint of the injected $\mathrm{HA}$. In rabbits the half-life of HA does not exceed one day and the clearance rates are considerably higher in OA than in normal rabbits. [19]. When injected inside the joint, $\mathrm{HA}$ is rapidly degraded, chiefly through various reactions with oxygen free radicals $[20,21]$. One can speculate that the short residence time of the drug into the joint may limit its diffusion within the target tissues. Decreasing the in situ HA degradation for optimizing clinical efficacy of viscosupplementation is a challenging research approach.

HAnox-M is a HA derivative, that combines a high concentration (35 $\mathrm{g} / \mathrm{l})$ of mannitol, with a non-cross-linked biofermentative HA. Mannitol is a polyol with radical oxygen species (ROS) scavenging properties [21]. Several in vitro studies have evidenced the effectiveness of mannitol to protect HA against ROS-mediated degradation [21-23]. The combination of mannitol to HA might extend the time of contact between HA and the target tissues and consequently might allow a more rapid onset of action than $\mathrm{HA}$ alone. However, the IA half-life of mannitol is likely too short $(<4 \mathrm{~h})$ to protect HA from degradation for several weeks and consequently to increase its duration of efficacy beyond the HA usual efficiency (6 to 12 months).

The HAV-2012 trial [24], a prospective, multicentre, randomized, non-inferiority study comparing HAnox-M to BioHA in patients with knee $O A$, has provided us the possibility to evaluate, through post-hoc analyses, whether the addition of mannitol is able to shorten the onset of action of viscosupplementation.

\section{Patients and Methods}

HAV-2012 study [24] was a prospective, double-blind, randomized, multicentre, parallel-group trial, conducted in in France between October 2012 and April 2014 (registration No. 2012-A00570-43). The aim of the trial was to compare both efficacy and safety of a 3 weekly injection regimen of HANOX-M (HAppyVisc ${ }^{\oplus}$, LABRHA SAS, Lyon, France), combining sodium hyaluronate (1-1.5 MDa, $31 \mathrm{mg} / 2 \mathrm{ml}$ ) with mannitol $3.5 \%$, to BioHA (Euflexxa ${ }^{\oplus}$, Ferring Pharmaceuticals, Parsippany, USA, 2.4-3.6 MDa, $20 \mathrm{mg} / 2 \mathrm{ml}$ ), in patients with symptomatic knee $\mathrm{OA}$. The study was performed in compliance with the principles of Good Clinical Practice (GCP) and the Declaration of Helsinki concerning medical research in humans and the country-specific regulations. It was approved by the Ethics Committee of Lyon Sud-Est IV. Before enrolment, patients were required to sign an informed consent form which complied with the requirements of the International Conference on Harmonisation (ICH).

All details of the study design and inclusion criteria were published previously [24]. Briefly, the study included males and females, aged $40-85$ years, fulfilling the ACR criteria for knee OA who failed to respond or were intolerant to analgesics and/or non-steroidal anti-inflammatory drugs (NSAIDs) or weak opioids and who had self-assessed their walking pain from 3 to 8 on a 11-point Likert scale $(0-10)$ at baseline. Bilateral knee X-rays were performed within the 3 previous months and included the following incidences: standing posteroanterior view, Lyon-schuss view, lateral view and skyline incidence of the patella. The OARSI score [25] for tibio-femoral (TF) joint space narrowing (JSN) was assessed on the radiological view highlighting the most severe lesions. The main exclusion criteria were OA flare, tibial plateau or femoral condyle bony attrition, excessive varus or valgus knee misalignment, viscosupplementation in the target knee within the previous 9 months, and IA corticosteroids use within the previous 3 months. Analgesics and ibuprofen (daily dose $\leq 800$ $\mathrm{mg}$ ) and naproxen (daily dose $<500 \mathrm{mg}$ ) were allowed. Symptomatic slow-acting drugs for OA were also allowed only if started at least 2 months before screening and not substantially altered during the study. NSAIDs at anti-inflammatory doses, strong opioids, systemic corticosteroids, IA corticosteroids and viscosupplements into the target knee were prohibited throughout the follow-up. Patients were asked to discontinue analgesic therapy 48 hours before each evaluation visit. Patients were randomized to one of the following treatment groups: HAnox-M or Bio-HA in a 1:1 ratio by blocks of 4 treatments, balanced 2:2.

Both viscosupplements were supplied in $2 \mathrm{ml}$ syringes containing $2 \mathrm{ml}$ of HA solution and were administered, one week apart, 3 consecutive weeks, into the target knee by an experienced physician who was unblinded to treatment and different from the clinical evaluator. The patient and the clinical evaluator were both blinded to treatment throughout the follow-up. The primary efficacy outcome was the variation, between baseline and the last follow-up visit at week (W)26, in the WOMAC A pain sub-score [26] measured on a 5 point Likert scale (5 pt-LS: 0=none, 1=mild, 2=moderate, 3=evere, 4=extreme; total score ranging 0-20). Secondary efficacy outcomes were the change throughout the follow-up of the following criteria: walking pain, patient global assessment, and WOMAC total score. Clinical assessment was performed by the clinical evaluator, blinded to treatment, at baseline, at the time of each injection on W 1, W2, then at the follow-up visits at W12 and W26. WOMAC A was assessed at weeks 1, 2, 3, 12 and 26. Other scores were obtained only at baseline, W12 and W26. The number and percentage of improved patients at week 1 and 2 were also studied, as well as the level of improvement. Patients had to grade their improvement from 0 (none) to 3 (high). Improved patients (who responded 2 or 3 ) had to quantify the level of improvement (<25\%, 25-50\%, 50-75\% and $>75 \%)$. Analgesic consumption and adverse events were recorded at each visit.

Statistical analyses were performed, from the Intent-to-treat (ITT) population, using XLStat 2015 Addinsoft software. The homogeneity of the two treatment groups was evaluated using non-parametric tests. The normality of variables was assessed using Shapiro-Wilk test. If normality, they were evaluated by analysis of variance. If matched data, the analysis was complemented by an appropriate t-test. In case of nonnormality, a generalized nonlinear model was performed in addition to or replacement of non-parametric tests. For quantitative data analysis, a model of logistic regression was performed and was supplemented if necessary by the McNemar test or by Fisher's exact test for confirmation of the effect significance. $\mathrm{P}$ values $<0.05$ were considered as statistically significant. 


\section{Results}

Two-hundred-five patients constituted the ITT population including 103 patients in the HAnox-M group and 102 in the BioHA group. Characteristics of the patients at baseline were consistent with those expected. The 2 treatment groups were statistically comparable for all the studied items. Variations and absolute values of all the outcomes were similar for both group at baseline, month 3 and end-point [24].

The median (range) of the WOMAC pain sub-score was 9.0 (4-19), 6.0 (0-19) and $5.0(0-15)$ in the HAnox-M group at baseline, W2 and W3 respectively. It was 9.0 (2-18), 7.0 (0-16) and 6.0 (0-14) in the BioHA group, namely a decrease of 1 more point in favor of HAnox-M, obtained from as soon as one week after the 1st injection. At month 3 and 6 the results were identical for both groups (Median=5.0 and 4.0 respectively). Despite this trend to a quicker efficacy of HAnox-M, the difference did not reach the statistical significance $(P=0.2)$. At W3, 76 patients (74.5\%) of the HAnox-M group and 65 (65\%) of the BioHA group, answered "good" or "very good" to the question "Have you experienced improvement since the first injection"? The patient's self-assessed percentage of improvement was $>50 \%$ in 30 and 23 patients, and $>75 \%$ in 8 and 3 patients for HAnox-M and BioHA patients respectively.

In subjects with the more severe joint space narrowing (OARSI grade $3 ; n=84$ ) the average decrease of pain (SD) was greater in patients treated with HAnox-M than in those treated with Bio$\mathrm{HA}$ at both W2: -3.0 (3.6) versus -1.9 (3.1), $\mathrm{P}=0.10$ and W3:-4.2 (3.2) versus -2.8 (2.6), $\mathrm{P}=0.048$ (Table 1 ).

Table 1: Variations of WOMAC A in patients with OARSI joint space narrowing grade 3.

\begin{tabular}{|c|c|c|c|c|c|}
\hline & & ITT Population & Hanox-M & $\mathrm{BioHA}$ & $P$ values \\
\hline \multirow{4}{*}{$\begin{array}{l}\text { WOMAC A variation } \\
\text { between D1 and D8 }\end{array}$} & $\mathrm{N}$ & 84 & 45 & 39 & \\
\hline & Missing (N) & 2 & 1 & 1 & \\
\hline & Mean (SD) & $-2.5(3.4)$ & $-3.0(3.6)$ & $-1.9(3.1)$ & \\
\hline & Median (Range) & $-2.0(12.5)$ & $-3.0(-12.5)$ & $-2.0(-12.4)$ & 0.1 \\
\hline \multirow{4}{*}{$\begin{array}{l}\text { WOMAC A variation } \\
\text { between D1 and D15 }\end{array}$} & $\mathrm{N}$ & 83 & 45 & 38 & \\
\hline & Missing (N) & 3 & 1 & 2 & \\
\hline & Mean (SD) & $-3.5(3.2)$ & $-4.2(3.6)$ & $-2.8(2.6)$ & \\
\hline & Median (Range) & $-3.0(-11.5)$ & $-4.0(-11.5)$ & $-3.0(-8.3)$ & 0.048 \\
\hline
\end{tabular}

\section{Discussion}

This post-hoc analysis of a controlled, randomized trial, showed a trend to an earlier reduction of pain with HAnox-M, compared to BioHA. Despite the present data cannot allow to conclude conclusively on that fact, one can draw hypotheses to explain our results.

Two recent in vitro studies, using powerful models of oxidative stress, showed that the addition of high concentration of mannitol to HA allowed to protect $\mathrm{HA}$ from degradation due to oxidant stress [22,23]. Mannitol (C6H14O6) is a polyol of molar mass $=182.17 \mathrm{~g} / \mathrm{moL}$, that demonstrated properties of reactive oxygen species scavenger. The beneficial effect of mannitol, due to its anti-oxidant power towards the rich reactive hydroxyl function, has been evidenced in several diseases [27-30]. During viscosupplementation, the interest to add mannitol to $\mathrm{HA}$ is obvious. Indeed, as soon as the viscosupplement is injected intra articularly, the HA macromolecular network, which contains many $\mathrm{OH}$ groups, reacts with ROS resulting in the rupture of the HA chains and accelerated degradation of the gel [20]. This rapid depolymerisation of HA is likely the primary cause for the short IA half-life of non-cross-linked HA. Another advantage of mannitol is its resistance to heat which permits sterilization by autoclaving, unlike other antioxidants such as polyphenols, vitamin C, vitamin E which are thermo labile and is hydro-soluble unlike antioxidants such as vitamin $E$ or betacaroten. Furthermore mannitol does not significantly alter the rheological behaviour of HA [23]. Polyols may also have direct effects on the osteoarthritic process, via their antioxidant activities. Cavone et al. [31] reported that topical mannitol applications on the hind paws of rats with adjuvant-induced arthritis reduced paw thickness and tissue oedema but not the inflammatory infiltrates. This anti-oedema effect of mannitol application occurred earlier than those prompted by a similar treatment with diclofenac or ketoprofen. In vitro a combination $\mathrm{HA}$ /sorbitol, isomer of mannitol, prevented IL-1 $\beta$-induced oxidative stress, stifled IL-1 $\beta$-induced metalloproteinase-13, nitric oxide (NO) and prostaglandin E2 release and NO synthase expression, attenuated cell death, caspase-3 activation and DNA fragmentation, in human chondrocytes [32]. Interestingly, the antioxidant as well as the anti-inflammatory and anti-catabolic effects of $\mathrm{HA}$ /sorbitol were attributed to sorbitol and $\mathrm{HA}$, respectively. Clinically, five open label studies assessing viscosupplements containing mannitol or sorbitol in patients suffering from knee [33-35] and hip [36,37] OA have been recently published. All showed a good ratio efficacy/safety. In a prospective randomized controlled trial the association crosslinked $\mathrm{HA}$ /mannitol was even shown to significantly reduce collagen II degradation compared to IA saline injection [38].

The present study suffers from some limitations mainly due to the fact that the trial was not designed for assessing accurately 
the early response to treatment. So, only WOMAC pain subscore and patient's self-assessment of efficacy were obtained at W1 and W2 and patients were not assessed between W2, date of the third injection, and month 3. Despite it has been possible, only one week after the second injection, to highlight an improvement $25 \%$ greater in the HAnox-M group ( -4 points versus -3 points), which allowed to reach the same level of relief to that obtained in the visit of the third months.

In summary, despite limitations which justify that further trials specifically designed for this purpose should be performed, our results suggest that mannitol might have beneficial effect on HA performances, by reducing pain more rapidly than HA alone, especially in patients with severe knee OA. The mechanisms by which mannitol could shorten the onset of action of viscosupplementation remain to be investigated.

\section{Acknowledgements}

The study was funded by LABRHA SAS, Lyon, France. The "HAppyvisc Study Group" included all investigators and coinvestigators of the study: Drs Ardizzone Marc, Bernard JeanPaul, Berthier Michel, Balblanc Jean-Charles, Bouysset Maurice, Caumon Nicolas, Charrin Jeanne, Colson Frederic, Despaux Jacques, Dusan Robert, Forestier Romain, Françon Alain, Gagnard Anne, Gerrud Sylvie, Guillemot Anne, Jalby Jocelyne, Kandil Franck, Laine Marc, Liens Daniel, Lohse-Walliser Anne, Mornand Yves, Noel Eric, Ollagnon Henri-Olivier, Perard David, Rambaud-Lequin Catherine, Rey Benedicte, Souchon Jean Francois, Toulemonde Gwendoline, Ferreira André, Markus Françoise, Camilleri Jean-Philippe, Milon Eric, Sparsa Laetitia, Tulli Regis, Bosque-Proton Annie, Legré-Boyer Virginie, Renzulli Jean-Georges, Ferrazzi Véronique, Roch-Bras Françoise, Monod Pierre, Roquefort Jean, Hacene-Beniddir Anissa, Fulpin Jean, Plantamura Alain, Cautiello Christian, Colcombet Clémence, Augé Benoit, Brault Isabelle, Letho-Gyselinck Hélène, Thomas Philippe, Gautier- Piquemal Marie-Charlotte

\section{Competing Interest:}

Thierry Conrozier: received fees from LABRHA SAS for scientific consultant services and study coordination. Naji Afif, Roger Lecurieux, Bernard Maillet, Jean-Charles Balblanc received honoraria from LABRHA SAS as clinical investigators of the study.

\section{Author's Contribution:}

TC: participated in the design of the study, was the national coordinator of the trial and wrote the manuscript.

FE: Wrote the manuscript and performed the statistical analyzis.

NA, BM, RL and MB: participated in the study and collected data as principal investigators of the trial.

XC participated in the design of the study and validated the final results as the president of the scientific committee.

All authors read, commented, made changes and then approved the final manuscript.

\section{References}

1. Jordan KM, Arden NK, Doherty M, Bannwarth B, Bijlsma JW, et al (2003) EULAR Recommendations 2003: an evidence based approach to the management of knee osteoarthritis: Report of a Task Force of the Standing Committee for International Clinical Studies Including Therapeutic Trials (ESCISIT). Ann Rheum Dis 62: 1145-1155.

2. Hochberg MC, Altman RD, April KT, Benkhalti M, Guyatt G, et al. (2012) American College of Rheumatology 2012 recommendations for the use of nonpharmacologic and pharmacologic therapies in osteoarthritis of the hand, hip, and knee. Arthritis Care Res 64: 465-474.

3. McAlindon TE, Bannuru RR, Sullivan MC, Arden NK, Berenbaum F, et al. (2014) OARSI guidelines for the non-surgical management of knee osteoarthritis. Osteoarthritis Cartilage 22: 363-388.

4. Bruyère, Cooper C, Pelletier JP Branco J, Luisa Brandi M, et al. (2014) An algorithm recommendation for the management of knee osteoarthritis in Europe and internationally: A report from a task force of the European Society for Clinical and Economic Aspects of Osteoporosis and Osteoarthritis (ESCEO). Semin Arthritis Rheum 44: 253-63.

5. Balazs EA, Denlinger JL (1993) Viscosupplementation: a new concept in the treatment of osteoarthritis. J Rheumatol 39: 3-9.

6. Balazs EA (2004) Viscosupplementation for treatment of osteoarthritis: from initial discovery to current status and results. Surg Technol Int 12: 278-289.

7. Wang CT, Lin YT, Chiang BL, Lin YH, Hou SM (2006) High molecular weight hyaluronic acid down-regulates the gene expression of osteoarthritis-associated cytokines and enzymes in fibroblast-like synoviocytes from patients with early osteoarthritis. Osteoarthritis Cartilage 14: 1237-1247.

8. Li P, Raitcheva D, Hawes M, Moran N, Yu X, et al. (2012) Hylan G-F 20 maintains cartilage integrity and decreases osteophyte formation in osteoarthritis through both anabolic and anticatabolic mechanisms. Osteoarthritis Cartilage 20: 1336-1346.

9. Li J, Gorski DJ, Anemaet W, Velasco J, Takeuchi J, et al. (2012) Hyaluronan injection in murine osteoarthritis prevents TGFbeta 1induced synovial neovascularization and fibrosis and maintains articular cartilage integrity by a CD44-dependent mechanism. Arthritis Res Ther 14: R151

10. Conrozier T, Balblanc JC, Richette P, Mulleman D, Maillet B, et al. (2012) Early effect of hyaluronic acid intra-articular injections on serum and urine biomarkers in patients with knee osteoarthritis: An open-label observational prospective study. J Orthop Res 30: 679-685

11. Henrotin Y, Chevalier X, Deberg M, Balblanc JC, Richette $P$, et al (2013) Early decrease of serum biomarkers of type II collagen degradation (Coll2-1) and joint inflammation (Coll2-1 NO ) by hyaluronic acid intra-articular injections in patients with knee osteoarthritis: a research study part of the Biovisco study. J Orthop Res 31: 901-907.

12. Wang Y, Hall S, Hanna F, Wluka AE, Grant G, et al. (2011) Effects of Hylan G-F 20 supplementation on cartilage preservation detected by magnetic resonance imaging in osteoarthritis of the knee: a two-year single-blind clinical trial. BMC Musculoskelet Disord 12 : 195.

13. Bannuru RR, Schmid CH, Kent DM, Vaysbrot EE, Wong JB, et al. (2015) Comparative effectiveness of pharmacologic interventions 
for knee osteoarthritis: a systematic review and network metaanalysis. Ann Intern Med 162: 46-54.

14. Miller LE, Block JE (2013) US-Approved Intra-Articular Hyaluronic Acid Injections are Safe and Effective in Patients with Knee Osteoarthritis: Systematic Review and Meta-Analysis of Randomized, Saline-Controlled Trials. Clin Med Insights Arthritis Musculoskelet Disord 6: 57-63.

15. Rutjes AW, Jüni $P$, da Costa BR, Trelle $S$, Nüesch E, et al. (2012) Viscosupplementation for osteoarthritis of the knee: a systematic review and meta-analysis. Ann Intern Med 157: 180-191

16. Vavken P, Dorotka R (2009) A systematic review of conflicting meta-analyses in orthopaedic surgery. Clin Orthop Relat Res 467: 2723-2735.

17. Campbell J, Bellamy N, Gee T (2007) Differences between systematic reviews/meta-analyses of hyaluronic acid/hyaluronan/ hylan in osteoarthritis of the knee. Osteoarthritis Cartilage 15: 1424-1436.

18. Bannuru RR, Natov NS, Obadan IE, Price LL, Schmid CH, et al. (2009) Therapeutic trajectory of hyaluronic acid versus corticosteroids in the treatment of knee osteoarthritis: a systematic review and meta-analysis. Arthritis Rheum 61: 1704-1711.

19. Lindenhayn K, Heilmann HH, Niederhausen T, Walther HU, Pohlenz K (1997) Elimination of tritium-labelled hyaluronic acid from normal and osteoarthritic rabbit knee joints. Eur J Clin Chem Clin Biochem 35: 355-363.

20. Stern R, Kogan G, Jedrzejas MJ, Soltés L (2007) The many ways to cleave hyaluronan. Biotechnol Adv 25: 537-557

21. Mendoza G, Alvarez Al, Pulido MM, Molina AJ, Merino G, et al. (2007) Inhibitory effects of different antioxidants on hyaluronan depolymerization. Carbohydr Res 342: 96-102.

22. Conrozier T, Mathieu P, Rinaudo M (2014) Mannitol allows to preserve the elasto-viscous properties of hyaluronic acid in an in vitro model of oxidative stress. Rheumatology and Therapy 1: 45-54.

23. Rinaudo M, Lardy B, Grange L, Conrozier T (2014) Effect of mannitol on hyaluronic acid stability in two in vitro models of oxidative stress. Polymers 6: 1948-1957.

24. Conrozier T, Eymard F, Afif N, Balblanc JC, Legré-Boyer V, et al . (2016)Happyvisc Study Group. Safety and efficacy of intra-articular injections of a combination of hyaluronic acid and mannitol (HAnOX-M) in patients with symptomatic knee osteoarthritis: Results of a double-blind, controlled, multicenter, randomized trial. Knee S0968-0160: 30060-30066.

25. Altman RD, Hochberg $M$, Murphy WA Jr, Wolfe $F$, Lequesne $M$ (1995) Atlas of individual radiographic features in osteoarthritis. Osteoarthritis Cartilage 3: 3-70.

26. Bellamy N, Buchanan WW, Goldsmith CH, Campbell J, Stitt LW (1988) Validation study of WOMAC: a health status instrument for measuring clinically important patient relevant outcomes to antirheumatic drug therapy in patients with osteoarthritis of the hip or knee. J Rheumatol 15: 1833-1840.
27. Belda JI, Artola A, García-Manzanares MD, Ferrer C, Haroun HE, et al. (2005) Hyaluronic acid combined with mannitol to improve protection against free-radical endothelial damage: experimental model. J Cataract Refract Surg 31: 1213-1218.

28. Magovern GJ, Bolling SF, Casale AS, Bulkley BH, Gardner TJ (1984) The mechanism of mannitol in reducing ischemic injury: hyperosmolarity or hydroxyl scavenger? Circulation 70: 91-95.

29. Suzuki J, Imaizumi S, Kayama T, Yoshimoto T (1985) Chemiluminescence in hypoxic brain- The second report: Cerebral protective effect of mannitol, vitamin E and glucocorticoid. Stroke 16: 695-700.

30. Fu W, Jiao X (1995) The effect of mannitol and anisodim on the prevention of free radical injury to post-ischemia flaps: an experimental study. Br J Plast Surg 48: 218-221.

31. Cavone L, Calosi L, Cinci L, Moroni F, Chiarugi A (2012) Topical mannitol reduces inflammatory edema in a rat model of arthritis. Pharmacology 89: 18-21.

32. Mongkhon JM, Thach $M$, Shi $Q$, Fernandes JC, Fahmi $H$, et al. (2014) Sorbitol-modified hyaluronic acid reduces oxidative stress, apoptosis and mediators of inflammation and catabolism in human osteoarthritic chondrocytes. Inflamm Res 63: 691-701.

33. Borras-Verdera, Calcedo-Bernal V, Ojeda-Levenfeld J, Clavel-Sainz $C$ (2012) Efficacy and safety of a single intra-articular injection of $2 \%$ hyaluronic acid plus mannitol in knee osteoarthritis over a 6month period. Rev Esp Cir Ortop Traumatol 56: 274-280.

34. Conrozier T, Bozgan AM, Bossert M, Sondag M, Lohse-Walliser A, et al. (2016) Standardized follow-up of patients with symptomatic knee osteoarthritis treated with a single intra-articular injection of a combination of cross-linked hyaluronic acid and mannitol. Clin Med Insights Arthritis Musculoskelet Disord 9: 1-5.

35. Heisel C (2013) Kipshoven. Safety and efficacy findings from a noninterventional study of a new hyaluronic acid/sorbitol formulation (Go-on ${ }^{\circledR}$ Matrix) for intra-articular injection to relieve pain and disability in osteoarthritis patients. Drug Res pp 445-449.

36. Conrozier T, Bossert M, Balblanc JC, Sondag M, Walliser-Lohse A (2014) Viscosupplementation with HAnox-M-XL is effective in moderate hip osteoarthritis but is not an alternative to hip joint surgery in patients with severe disease. results of a clinical survey in 191 patients treated in daily practice. EMJD 3: 49-55.

37. Migliore A, Massafra U, Bizzi E, Tormenta S, Cassol M, et al. (2014) Duration of symptom relief after intra-articular injection of hyaluronic acid combined with sorbitol (anti-ox-vs) in symptomatic hip osteoarthritis. Int J Immunopathol Pharmacol 2: 245-252.

38. Henrotin $\mathrm{Y}$, Berenbaum $\mathrm{F}$, Chevalier $\mathrm{X}$, Marty $\mathrm{M}$, Richette $\mathrm{P}$, et al. (2015) Significant reduction of the Serum Levels of a Specific Biomarker of Cartilage Degradation (serum Coll2-1) following Viscosupplementation Compared to Placebo in patients with Knee Osteoarthritis: the EPIKART Study. Topics di Terapia IntraArticolare 7: 21-22. 